\title{
Quality and Reliability of LES of Convective Scalar Transfer at High Reynolds
}

\section{Numbers}

Qi Li , Elie Bou-Zeid ${ }^{1 *}$, William Anderson ${ }^{2}$, Sue Grimmond , Marcus Hultmark ${ }^{4}$

1 Department of CEE, Princeton University, US

2 Department of Mechanical Engineering, University of Texas at Dallas, US

3 Department of Meteorology, University of Reading, UK

4 Dept. of Mechanical and Aerospace Engineering, Princeton University, US

* Corresponding author at: CEE-EQuad-E414, Princeton University, Princeton, NJ 08544, USA. ebouzeid@princeton.edu

\section{Abstract}

Numerical studies were performed to assess the quality and reliability of wall-modeled large eddy simulation (LES) for studying convective heat and mass transfer over bluff bodies at high Reynolds numbers $(R e)$, with a focus on built structures in the atmospheric boundary layer. Detailed comparisons were made with both wind-tunnel experiments and field observations. The LES was shown to correctly capture the spatial patterns of the transfer coefficients around two-dimensional roughness ribs (with a discrepancy of about $20 \%$ ) and the average Nusselt number $(\mathrm{Nu})$ over a single wall mounted cube (with a discrepancy of about 25\%) relative to wind tunnel measurements. However, the discrepancy in $R e$ between the wind tunnel measurements and the real-world applications that the code aims to address influence the comparisons since $\mathrm{Nu}$ is a function of $R e$. Evaluations against field observations are therefore done to overcome this challenge; they reveal that, for applications in urban areas, the wind-tunnel studies result in a much lower range for the exponent $m$ in the classic $N u \sim R e^{m}$ relations, compared to field 
measurements and LES $(0.52-0.74$ versus $\approx 0.9)$. The results underline the importance of conducting experimental or numerical studies for convective scalar transfer problems at a $R e$ commensurate with the flow of interest, and support the use of wall-modeled LES as a technique for this problem that can already capture important aspects of the physics, although further development and testing are needed.

\section{Introduction}

Convective heat and mass transfer at high Reynolds numbers $\left(\operatorname{Re} \sim 10^{6}-10^{8}\right)$ over complex surfaces is of interest for many engineering and environmental applications, such as heat exchanger design, agricultural and urban meteorology, and building energy studies. The latter applications are of growing significance due to rapidly expanding urbanization interacting with global climate change to alter the urban environment and the resource intensity of cities in complex ways. The convective heat transfer coefficient over the exterior surfaces of buildings is a key parameter for modeling the exchange of energy between buildings and their environment. This exchange needs to be quantified to calculate accurate heating and cooling loads $[1,2]$, to assess the energy performance of the building envelope [3], and to better simulate the urban environment under a changing climate [4].

In addition, with the heat-mass transfer analogy [5], knowledge on the turbulent transfer of temperature (under conditions where it can be considered as a passive scalar) is 
transferable to studies on the exchange of other scalars, especially carbon dioxide and moisture [6], which are important for example for assessing the performance of green roofs [7,8]. For urban climatological and meteorological studies, it is crucial to simultaneously capture the turbulent heat and water vapor surface fluxes, which are typically parameterized through an urban canopy model (UCM) [9-12] in coarse geophysical simulations. The transfer coefficients for heat and water vapor are important parameters in these UCMs [13], but their current parameterizations are partially based on experimental results that are over 90 years old [14]. Improved parameterizations would involve environmental turbulent boundary layer flows over large roughness elements the height of which can be a significant fraction of the total boundary layer depth. Such surfaces are termed very rough in Castro et al.[15] and the resulting flow differs from the classic rough-wall boundary layers discussed for example in Jiménez [16] where the height to boundary layer depth ratio is limited to be below 0.025. Advancing our understanding of the fundamental transport processes of heat and moisture over such complex surfaces, and how to model them via transfer coefficients beyond the current state of the science, is hence urgently required in view of the wide range and importance of the related applications.

Three different approaches have been traditionally taken to gain a better understanding of the convective transfer coefficients. The first approach is placing scale models in wind tunnels and measuring the convective transfer of either some substance or temperature, 
while minimizing the effect of buoyancy (which could nonetheless be quite important in real urban terrain). These studies [17-24] often considered cases at lower Reynolds numbers $\left(10^{3}-10^{4}\right)$ (due to length scale limitations), with a developing turbulent boundary layer in a parallel channel flow. Mass transfer experiments, usually with Naphthalene sublimation techniques $[18,25]$ or water evaporation [23], were performed to study the mass transfer from surface-mounted cubes in a wind tunnel. These are only some examples of wind tunnel studies from the extensive literature, which was summarized in relatively recent reviews $[2,3]$. One advantage of wind tunnel studies is that the spatial variation of heat/mass transfer coefficients along the surfaces of the bluff elements can be accurately measured. The setup of the experiments can also be varied to investigate the effects of different angles of attack [19] and geometric configuration of the roughness elements [17,23], among other topographically complexities. However, a simple extension of these studies to the environment has to be handled with caution. The Reynolds number of the typical atmospheric boundary layer (ABL) is 3-4 orders of magnitude higher than that of common wind tunnels. Unlike momentum exchange, which is fully dominated by form/pressure drag over complex topographies at high $R e$, heat and mass exchanges are always performed by molecular conduction or diffusion in the vicinity of the complex interface and do not lose their dependence on the molecular heat and mass diffusivities at high $R e$. Neither the convective to conductive/diffusive scaling represented by the Nusselt number for heat $(\mathrm{Nu})$ or Sherwood number for mass $(\mathrm{Sh})$, nor 
the inertial scaling given by the Stanton number $(S t \sim N u / R e)$, become independent of $R e$ in general (See Lienhard and Lienhard[26]). Re-independence for $S t$ might be approached or expected only if the flow over each facet is itself also fully rough [27], which is not always the case over urban terrain since the surfaces of building facets might be smooth or transitional. The empirical correlations of $N u$, Sh, or St with Re obtained from these scale model experiments are thus not directly applicable to heat or mass transfer from buildings [23]. In addition, the usually thin inflow turbulent boundary layers [2] and the low turbulent intensity levels are further reasons why wind tunnel studies of heat and mass transfer, although providing very valuable insight, have limitations that preclude the direct application of their findings to large scale flows at high $R e$, such as flows in the real natural environment [28].

Another approach that overcomes the problem of low $R e$ in wind tunnel studies is full-scale experiments conducted outdoors on buildings or structures [29-33]. These field experiments give very valuable information especially on the correlation between the heat transfer coefficient and wind speed, which can be generalized to a power-law relation between $\mathrm{Nu}$ and $\mathrm{Re}$. One manifestation of the continued dependence of heat and mass exchange on $R e$ is that the exponents in such power laws are themselves $R e$ dependent, and thus these empirical relations apply only in the range of $R e$ in which they were developed. From the perspective of modeling, such full-scale field-derived empirical relations are therefore useful for both building energy simulations and urban 
climate studies [1,13]. However, generalization of the findings can also be challenging due to the influence of the exact shapes of the building facets, the texture/roughness of the building surface materials, and the surrounding structures in the outdoor environment. In addition, the positions at which the temperature and wind velocity are measured vary across different field studies, further complicating inter-comparisons between them to extract more universal empirical relations.

Numerical simulations are another useful methodology to study this problem. Reynolds averaged Navier-Stokes (RANS), large-eddy simulations (LES) or direct numerical simulations (DNS) have been carried out in the recent years to study the turbulent transfer of momentum and scalars over rough surfaces with roughness elements that mimic buildings or urban canyons [34-37]. Since the computational cost of resolving the viscous layer (i.e. DNS [38-41] or wall-resolved LES [42]) is too high for applications at $R e$ commensurate with the real-world (limiting these techniques to low $R e$ where the same challenges discussed above for wind tunnels reemerge), wall modeling is often adopted for RANS or wall-modeled LES studies. The 'law of the wall' or related equilibrium approaches, which are based on the concept of universal behavior of momentum and scalars in the inertial (logarithmic) layer, are often adopted [34,35,43-45]. These types of wall models have some known caveats in complex flow regions [46]; however, good agreement of models using such equilibrium laws with experiments have been found by both Park et al. [34] and Liu et al. [44] in their studies of transfer of scalars over 
geometrically complex surfaces. The application of such equilibrium wall-models in LES pose additional challenges (compared to RANS) that were very comprehensively assessed by Wyngaard et al. [47]. Various other more sophisticated wall-models that should in principle offer better performance have been proposed such as models that solve the boundary layer equations numerically [48] or analytically [49,50], or models that use a "customized temperature wall function" (CWF) (though based on low Reynolds number results) [51]. Nevertheless, the challenge of wall-modeling in LES remains open [52,53], even when the very important influence of buoyancy and how to represent it correctly in wall models (particularly for vertical walls) is ignored. This challenge frames the scope and goals of this paper.

Given that for studies of turbulent flow and transport over urban-like rough surfaces at high $R e$ wall-modeled LES is a feasible and very appealing tool, there is a growing urgent need to assess its skill in capturing turbulent scalar transport. The near-surface performance is more critical for scalars than for momentum (again due to the dominance of form drag, which is partially resolved in LES, for momentum), and as such the role of the wall-model is more prominent. But if the shortcomings of current wall models can be investigated, quantified, and potentially overcome, the impact on future studies that focus on scalar transport under high Re scenarios can be substantial. It is worthwhile to stress again the importance of studying the heat/mass transfer problem at a Reynolds number that is representative of the real problem of interest (which is possible with wall-modeled 
LES), given that the scalar transfer is inherently Re-dependent.

Therefore, the objective of this study is to provide a thorough assessment of wall-modeled LES by detailed comparisons to both scale-model and full-scale studies. Knowing the capabilities and limitations of this numerical approach will help to draw more sensible conclusions for future applications in building energy and urban climatology studies. A practical question we seek to answer is: are the errors resulting from the parameterization of unresolved scales (wall and subgrid scale models) in LES larger or smaller than the errors involved in extrapolating from low-Re approaches (DNS or wind tunnels) to high-Re real world flows, for scalar transfer problems?

This paper is organized as follows: section two describes the numerical details of the large eddy simulation; section three discusses the comparison of the local scalar transfer coefficient with wind-tunnel studies of two-dimensional roughness; section four considers both the local and average transfer coefficients by comparing to wind-tunnel studies of a single cube; section five focuses on the comparison with full-scale field measurement, section six provides a summary and conclusions.

\begin{tabular}{|l|l|l|l|}
\hline \multicolumn{4}{|l|}{ Nomenclature } \\
\hline$c_{p}$ & specific heat at constant pressure & $z_{0 s}$ & scalar roughness length \\
$h_{c}$ & heat/mass transfer coefficient & $x, y, z$ & $\begin{array}{l}\text { streamwise, cross-stream and vertical } \\
\text { coordinate }\end{array}$ \\
& $q_{s} /\left(s_{0}-s_{r e f}\right)$ & & heat conductivity of solid surface \\
$H$ & height of the obstacle (rib or cube) & $\lambda$ & \\
\hline
\end{tabular}




\begin{tabular}{|c|c|c|c|}
\hline$L_{\mathrm{i}}$ & LES domain size in direction $i$ & \multicolumn{2}{|c|}{ Subscripts } \\
\hline$m$ & power exponent in $\mathrm{Nu}-\mathrm{Re}$ relation & $x, y, z$ & streamwise, cross-stream and vertical \\
\hline $\operatorname{Re}$ & Reynolds number $=u H / v$ & & directions \\
\hline$s$ & scalar concentration & LES & quantities from LES \\
\hline$u$ & characteristic velocity scale & $\operatorname{Exp}$ & quantities from experiments \\
\hline$u *$ & Friction velocity $=\left(-\tau_{w}\right)^{1 / 2}$, where $\tau_{w}$ is & 0 & quantity at surface \\
\hline & the total kinematic wall shear stress & ref & quantity at reference height \\
\hline$z 0 m$ & momentum roughness length & & \\
\hline
\end{tabular}

\section{Wall-modeled LES and Dynamic Roughness Wall Model}

The LES code uses the immersed boundary method (IBM) to account for presence of the roughness elements, in which a discrete time momentum forcing is used to simulate the immersed boundary force $[54,55]$. The filtered incompressible continuity, Navier-Stokes and scalar conservation equations (Eq.1-3, respectively) are solved assuming hydrostatic equilibrium (we will omit the usual tilde above the variables that denotes filtering for simplicity, but all the variables we will discuss are the filtered/resolved components solved for in LES unless otherwise noted)

$$
\begin{gathered}
\frac{u_{i}}{x_{i}}=0 \\
\frac{\partial u_{i}}{\partial t}+u_{j}\left(\frac{\partial u_{i}}{\partial x_{j}} \frac{\partial u_{j}}{\partial x_{i}}\right)=\frac{\partial p}{\partial x_{i}} \frac{\partial{ }_{i j}}{\partial x_{j}}+F_{i}+B_{i} \\
\frac{s}{t}+u_{i} \frac{s}{x_{i}}=\frac{q_{i}^{s}}{x_{i}},
\end{gathered}
$$


where $t$ denotes time; $u_{i}$ is the resolved velocity vector; $p$ is the modified pressure; ${ }_{i j}$ is the deviatoric part of the subgrid stress tensor; $F_{i}$ is the body force driving the flow (here simply a homogeneous steady horizontal pressure gradient along the $x$ direction); and $B_{i}$ is the immersed boundary force representing the action of the obstacles (buildings) on the fluid. The density is assumed equal to 1 (all the equations are normalized so the numerical value of the density is irrelevant). In Eq.(3), $s$ denotes a passive scalar quantity and $q_{i}^{s}$ is the $i^{\text {th }}$ component of the subgrid scale scalar flux. Although the code can simulate active scalars (see [56,57]), the experimental data we identified for code evaluation were under conditions where buoyancy played an insignificant role.

The code uses a pseudo-spectral method for computing the horizontal spatial derivatives on a uniform staggered Cartesian grid. To overcome the Gibbs phenomenon that emerges from the combined application of the IBM method with spectral derivatives, a smoothing approach we developed and detailed in $\mathrm{Li}$ et al. [58] is adopted. Vertical spatial derivatives are obtained from second-order centered finite difference. Second order Adams-Bashforth time integration is used. The subgrid scale (SGS) stress tensor is modeled using the Lagrangian scale-dependent dynamic Smagorinsky model [59], while the SGS scalar flux model uses the dynamically computed SGS viscosity with a constant SGS Prandtl number $\left(\operatorname{Pr}_{S G S}\right)$ of 0.4 (this is unrelated to the molecular $\left.\operatorname{Pr}[60]\right)$.

In this study, we adopt a new approach for dynamically evaluating the momentum and scalar roughness lengths in the expression of the log-law wall model. The general log-law 
wall model for momentum and scalars is given by:

$$
\begin{gathered}
\frac{u}{u_{*}}=\frac{1}{-} \log \left(\frac{z}{z_{0 m}}\right), \\
\frac{s_{0} \quad s}{S_{*}}=\frac{1}{-} \log \left(\frac{z}{z_{0 s}}\right),
\end{gathered}
$$

where $u$ is the local wall-parallel velocity near the wall; $s_{0}$ is the scalar concentration or temperature at the surface; $u *$ is the friction velocity calculated as the square root of the kinematic wall shear stress $\tau_{w} ; s *$ is the mass flux concentration or heat flux temperature (defined as the kinematic surface flux divided by $u *$ ); $z$ is distance away from the wall in the wall-normal direction; $\kappa=0.4$ is the von Kármán constant; and $z_{0 m}$ and $z_{0 s}$ are the roughness lengths for momentum and scalars, respectively. These roughness lengths are often chosen according to the roughness types of the surfaces for hydrodynamically rough walls. However, building facets are often hydrodynamically smooth, including the experiments we compare to. Therefore, instead of adopting a fixed roughness, we dynamically model the roughness lengths for momentum and scalars as a function of the viscous length scale $v / u *$. In fact, it has been shown by Kader and Yaglom [61] that similar reasoning to the one that yielded the Prandtl-Nikuradse momentum skin friction law for smooth pipe and channel flow can be applied to scalar transfer in a turbulent flow to obtain heat or mass transfer laws for a smooth wall, with some unknown quantities that can be determined from experiments. Eq.(3) can be rewritten following the Prandtl-Nikuradse skin friction law as 


$$
\left(\frac{u}{u_{*}}\right)=A \log \left(\frac{z}{/ u_{*}}\right)+B
$$

which can be further rearranged into

$$
u_{*}=\frac{u}{A \log \left(\frac{z e^{B / A}}{/ u_{*}}\right)}=\frac{u}{A \log \left(\frac{z}{z_{0 m}}\right)},
$$

where $A$ and $B$ are determined from experiments and $z 0 m$ is given by

$$
z_{0 m}=\frac{-}{u_{*}} e^{B / A}
$$

The same dimensional analysis can then be similarly developed for scalars:

$$
s_{0} \quad s(z)=s_{*} \quad\left(u_{*} z /, /\right)
$$

where $\chi$ is the mass or thermal diffusivity, and $\psi$ is a dimensional analysis function to be determined empirically (with the aid of profile-matching as for velocity). Eq.(3) is a general one for turbulent mass or heat transfer in wall-bounded flows. For air, $\operatorname{Pr}=0.7$ and $s_{*}=q_{s} /\left(c_{p} u_{*}\right)$, where $q_{s}$ is the dynamic heat flux at the wall and $c_{p}$ the heat capacity of the air. The experiments to determine the form of Eq.(3), as detailed in Kader and Yaglom [61], then yield the log-law for scalar:

$$
\frac{s_{0} \quad s(z)}{S_{*}}=\log \left(\frac{z}{/ u_{*}}\right)+.
$$

For air, $\alpha$ and $\beta$ can be found from experiments for heat transfer with weak buoyancy. If $s$ represents air temperature, then the heat flux at the wall is given by 


$$
\frac{q_{s}}{c_{p}}=u_{*} s_{*}=u_{*} \frac{\left(s_{0} s(z)\right)}{\log \left(\frac{z e^{\prime}}{/ u_{*}}\right)}=u_{*} \frac{\left(s_{0} s(z)\right)}{\log \frac{z}{z_{0 s}}},
$$

where $z_{0}$ for the scalar can be written as:

$$
z_{0 s}=\frac{-}{u_{*}} e^{\prime}
$$

The roughness length expressions in Eq.(3) and Eq.(3) should be universal for smooth walls, and thus we can adopt the constants determined by Kader and Yaglom from experiments for fully turbulent flows [61,62] (Table 1 in Kader and Yaglom[61]; $A$ can be viewed as the inverse of the von Kármán number, but only the ratios $B / A$ and $\beta / \alpha$ influence the results and here we select the same ratio of 3.9/1.8 for both momentum and scalars, which effectively yield

$$
z_{0 m}=z_{0 s}=\frac{v}{8.73 u_{*}} \simeq \frac{v}{9 u_{*}}
$$

This result applies for molecular Prandtl of Schmidt numbers $\sim 1$, which is a reasonable approximation for all the tests we conduct in this study. These length scales depend on $u *$ which varies in space and time over complex geometries. We thus use an explicit approach where $u *$ form the previous time step is used in Eq. (3) to determine $z 0 m$ at every wall location, and then the updated $z_{0 m}$ is used to compute $u *$ from Eq.(3). This dynamic equilibrium wall-model controls the fluxes at the solid-fluid interface, and therefore is important to determine if the LES is able to capture the physics of the flow and reproduce experimental observations. It is important to note here that this model, by construction 
since it assumes smooth facets, yields a Stanton number that is $R e$ dependent. On the other hand, if the facets were assumed fully rough with constant $z o m$ and $z o s$, the heat transfer regime would become $R e$ independent. We assume the presence of a logarithmic form at the first grid point away from the wall of the solid, which is commonly done in direct forcing immersed boundary method as adopted here.

\section{Spatial variation of the transfer coefficient compared to a wind tunnel study}

\subsection{Experimental setup of mass transfer over two-dimensional ribs}

The dimensional (e.g. in $\mathrm{W} \mathrm{K} \mathrm{K}^{-1} \mathrm{~m}^{-2}$ ) local heat or mass transfer coefficient is defined as

$$
h_{c}=\frac{q_{s}}{s_{0} \quad s_{r e f}} \text {, }
$$

where $s_{r e f}$ is some reference scalar quantity in the fluid. The distributions of the local heat and mass transfer coefficients obtained from detailed scale-model measurements have large spatial variations over the surface of roughness elements due to the highly complex flow patterns involving separations and reattachments in the flow. It is therefore desirable to assess the capability of the wall-modeled LES in predicting these spatial patterns of local heat and mass transfer coefficients.

Nevertheless, one here again faces the challenge that the magnitudes of $h_{c}$ in scaled-model experiments at lower $R e$ and LES at larger $R e$ are not directly comparable due to the dependence of $h_{c}$ on $R e$. However, since the momentum dynamics are less 
sensitive to $R e$, the spatial flow patterns should match as long as the scaled-model $R e$ exceeds $\sim 10^{5}$, and therefore the resulting spatial variation patterns of $h_{c}$ should be comparable. Therefore, to overcome the magnitude discrepancy and still compare the spatial variabilities, the heat or mass transfer coefficients from different scale-model experiments and numerical simulations are usually normalized for appropriate comparison [13].

The measurement of mass transfer coefficient from a wind-tunnel study on evaporation of water from two-dimensional roughness (ribs) by Narita [23] is used here as a benchmark case to assess the LES. The roughness elements, made of acrylic resin of $1 \mathrm{~mm}$ thickness, were covered with wetted filter paper. A fine thermistor sensor was inserted just below the paper surface to monitor the surface temperature. The evaporating surface is assumed to be at saturation. A weighing method was used to obtain the evaporation rate and thus the mass transfer coefficient can be estimated by knowing the ambient water vapor concentration. Measurements were conducted at a low relative humidity to keep the experimental error of the transfer coefficient to within $4 \%$.

Note that the sharp edges of these 2D ribs fix the separation points to the downstream top corners of each rib, and thus strengthen the insensitivity of the flow patterns to $R e$ and improve the flow simulation results [63]. 


\subsection{Numerical model of mass transfer}

We considered configurations with three different separation distances between the two-dimensional ribs. Figure 1 is a side view of the basic configuration. The rib height $H$ is represented with 16 grid points. We use a horizontally periodic boundary condition for momentum and mass (thus we are simulating infinite repetitions of the patterns shown in Figure 1). The longer section behind the ribs is used to ensure that the inflow velocity at the first rib is free of the wake influence from the fifth element. It also mimics the test section surface upstream of the ribs in the open circuit wind tunnel [23]. The experimental Reynolds number is 16000 , where velocity is fixed at $4 \mathrm{~m} / \mathrm{s}$ at the top of the boundary layer and length scale is the rib height. The experiment did not precisely control the humidity in the incoming air in the wind tunnel. Instead, during each run where the evaporation rate was measured, the evaporation rate from a flat plate placed in the free stream was simultaneously recorded for normalizing the measurements. Therefore, we could not replicate the exact details of the mass inflow, but again these only affect the magnitude and not the spatial patterns of the transfer coefficient that we seek to investigate here. 


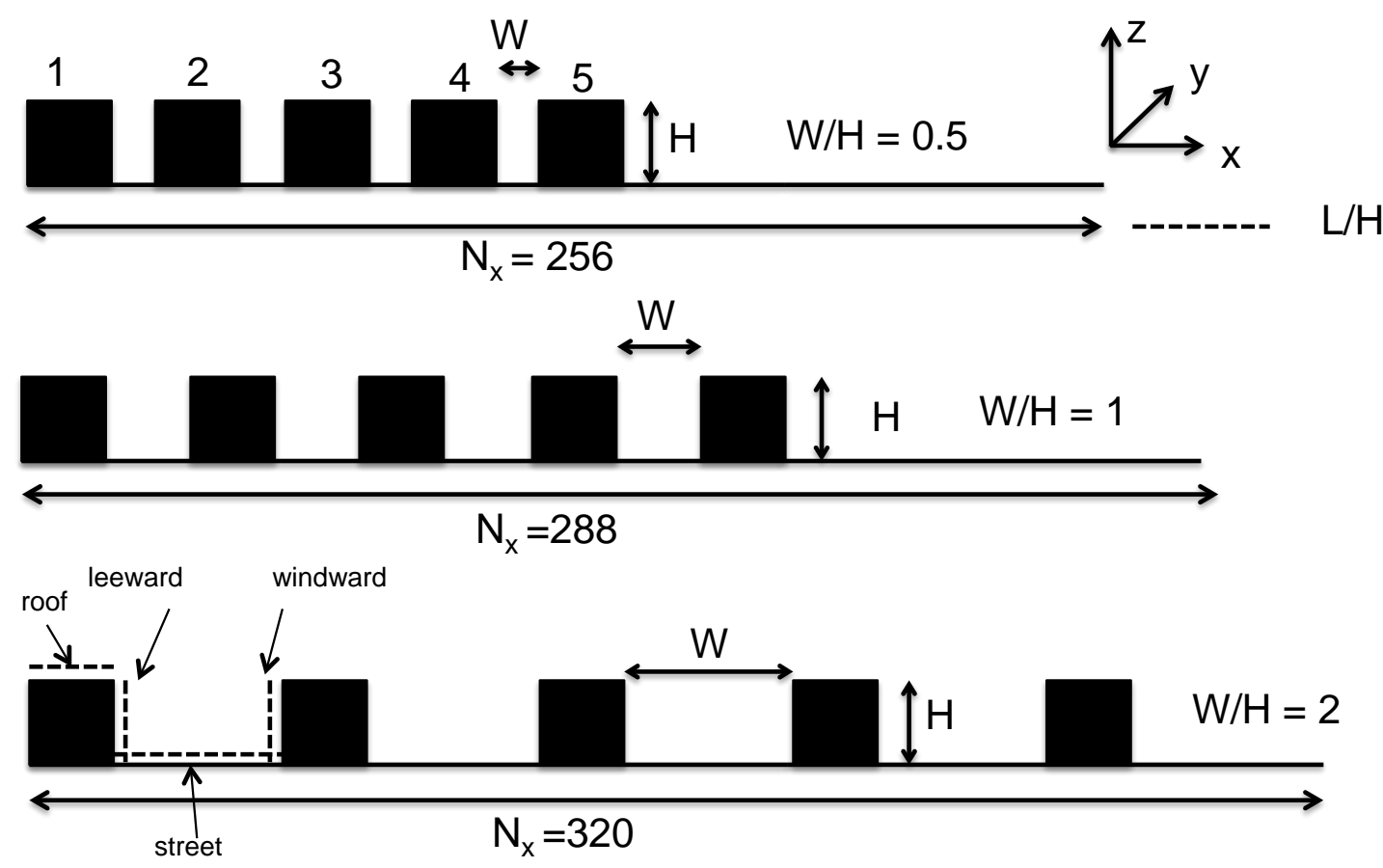

Fig. 1. Side view of the geometric configuration of the numerical simulations. The cases of $W / H=0.5,1$ and 2 are shown in the figure from top to bottom. Inflow is from left to right. $N_{x}$ is the number of grid points in $x$-direction. $N_{z}=80$ total vertical grid points for all three cases.

The top boundary condition in the simulation is slip-free for momentum and zero-flux for the scalar (same top BC for all simulations in this paper). The dimensions of the wind tunnel are $0.9 \mathrm{~m}$ in height and $1.8 \mathrm{~m}$ in width. The height of the wind tunnel is 15 times the height of the rib $H=0.06 \mathrm{~m}$ We have conducted preliminary tests by varying the domain height from 3 times to 10 times $H$ (results not shown here) to test the sensitivity to the domain height. We found that results with domains exceeding $5 H$ in height converge, and therefore we adopt $5 H$ as our domain height in all simulations in this 
section. The boundary condition on the surfaces of the ribs for water vapor is assumed to be at a constant concentration, which is justified by the saturated state of the wetted surfaces. All cases were run for about 20 eddy turn over times $\left(L_{z} / u *\right)$ and averaged in the $y$-direction, to reach statistical convergence, which was further confirmed by ensuring that the velocity profiles reach a steady state, i.e. they become invariable if the averaging time is further increased.
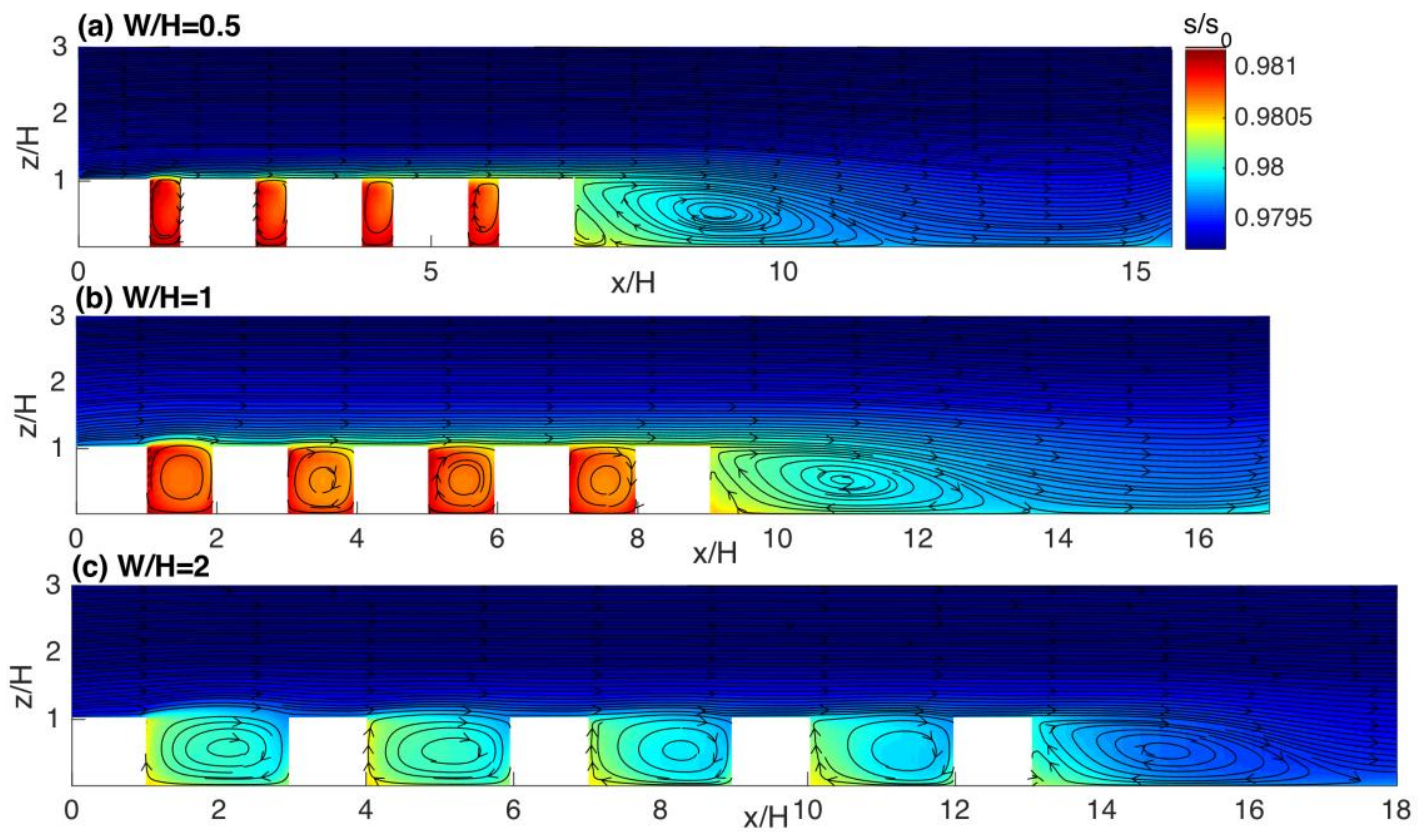

Fig. 2 Mean (time- and $y$-averaged) contour plots of $s / s_{0}$ and streamlines. The wind is from left to right. The white spaces represent the transect areas occupied by the solid 2-dimensional ribs. Color scale for the normalized scalar concentration is the same for all three cases.

Figure. 2 (a)-(c) shows the pseudocolor plots of the scalar concentration normalized by the surface scalar concentration, together with the streamlines. The central vortices in the 
$W / H=0.5$ and 1 cases are characteristic of the 'skimming flow' regime and explain the high concentrations of scalar in the space between the ribs ("the street canyon"), whereas the slightly asymmetric flow field in case $W / H=2$ is evidence of more complex flow interactions in the 'wake interference regime' $[64,65]$ that allows more exchange between the canyon and the air aloft. The flow patterns are consistent with the regime expected for this geometry. In addition to the more intensive exchanges for the widest canyon, the reduced "emitting surface" to "canyon volume" ratio, $(W+2 H) /(H W)=H+2 / W$, when $W$ increases and $H$ is maintained constant, further explains the reduced concentrations in the canyon.

Figure. 3 (a)-(c) shows instantaneous contour of the scalar concentration normalized by the surface scalar concentration, together with the streamlines along one $x z$-slice at a fixed $y$. The instantaneous structures in the scalar concentration field, as well as the streamlines, are generally distinct from their averaged counterparts shown in figure 2 , particularly for the $W / H=2$ case. The depicted turbulent structures are important for the vertical exchange; for example, one can observe the strong ejection from the last canyon in Figure 3(c) for the $W / H=2$ case. This is consistent with general observations for such kind of type-k roughness where the eddies of scale $H$ are shed out of the cavity, resulting in the more complex flow interactions. The instantaneous vortices inside the canyons for the two other cases, especially $W / H=1$ in $3(b)$, are somewhat more similar to their time 
and space averaged counterparts in figure 2(b). This dominant mean circulation inside the canyons for these cases might hinder ejections and sweeps near the top of the canyons and reduce the instantaneous exchange between canyons and air above. While we show only one snapshot here; other snapshots we analyzed conveyed the same information.

(a) $\mathrm{W} / \mathrm{H}=0.5$
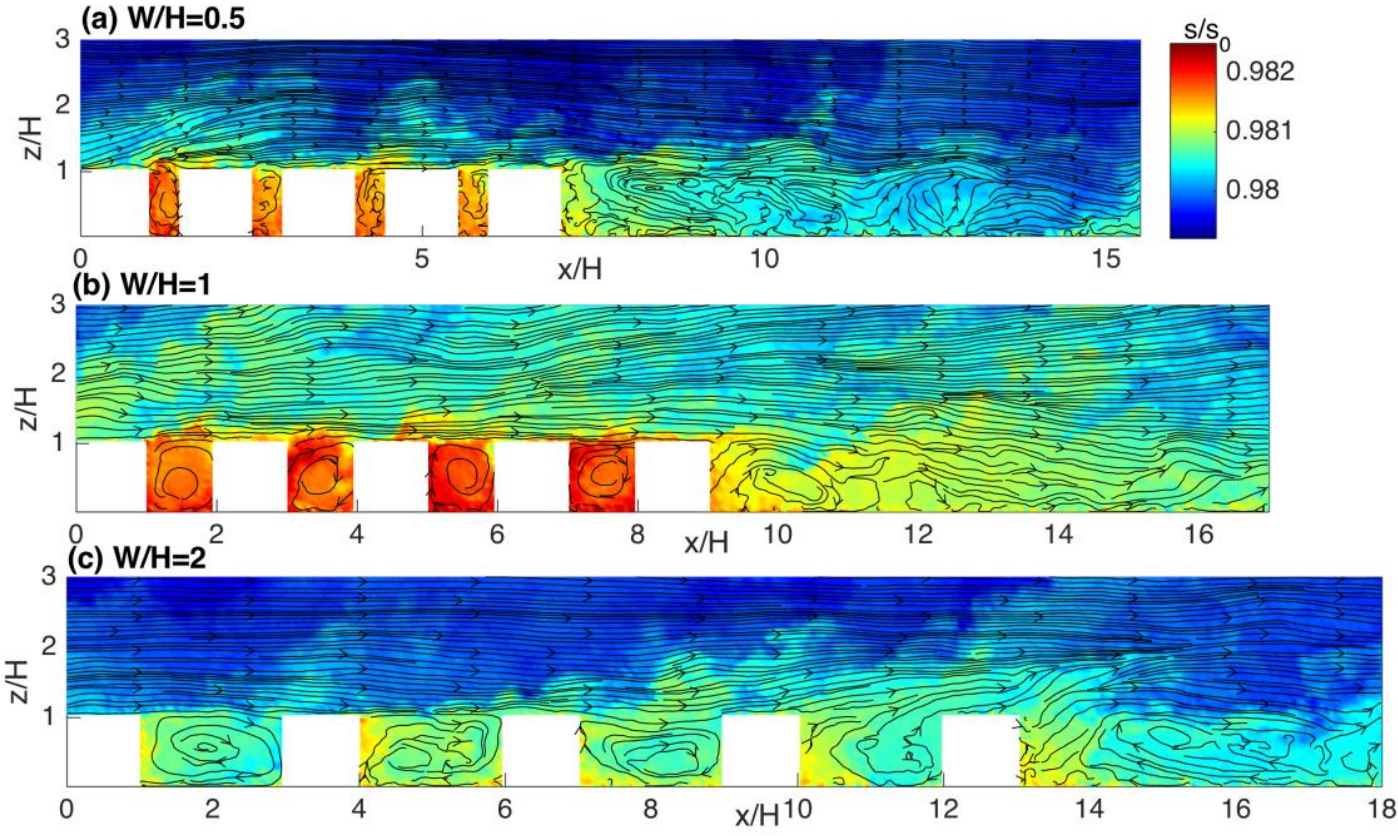

Fig. 3 Snapshots of instantaneous $s / s_{0}$ and streamlines. The wind is from left to right. The white spaces represent the transect areas occupied by the solid 2-dimensional ribs. Color scale for the normalized scalar concentration is the same for all three cases.

Figure. 4 shows the comparisons between the experimental and LES results for the three rib separations, while Table 1 lists the absolute percentage deviation of the LES from the experiments. All quantities are normalized by the average mass transfer coefficient on the floor in between two consecutive ribs. The experimental data are averaged over multiple 
ribs starting where the transfer coefficient over subsequent ribs converge. To best mimic the experimental data, we average the LES result using relevant quantities from the second to the fifth rib, where the transfer coefficients become independent of location of the ribs. We tested different averaging ranges and the impact on the results is minimal The resulting general spatial trends for each case, as well as the changes in transfer coefficient patterns as a result of the variation in the separation distance, are adequately captured by the LES. Despite the fact that the leeward transfer coefficient varies quite considerably across different cases, its variation is captured well: for example, the peak for $W / H=1$ was observed to occur at about $0.4 H$ from the bottom and this maximum is also clear in LES. Both the experiment and the LES also show that the decrease along that face at $W / H=0.5$ is more pronounced than $W / H=2$. The variation on the street face (floor between two ribs) is also reasonably captured by the LES. The maximum of the transfer coefficient on the street occurs at about $0.5 \mathrm{H}$ in the experiments for cases $\mathrm{W} / \mathrm{H}=1$ and $W / H=2$, which is also the location predicted by the LES. This peak matches the location of the highest wall-parallel velocity produced by the recirculating flow in the canyon. Given the complexity of the wakes and recirculation inside the canyon, the matching of the observed time-averaged transfer coefficients that are modulated by these flow patterns indicate that the wall-modeled LES is capable of reproducing them, as well as the spatial distributions of the local mass transfer they generate inside the canyon. 
(a) $\mathrm{W} / \mathrm{H}=0.5$

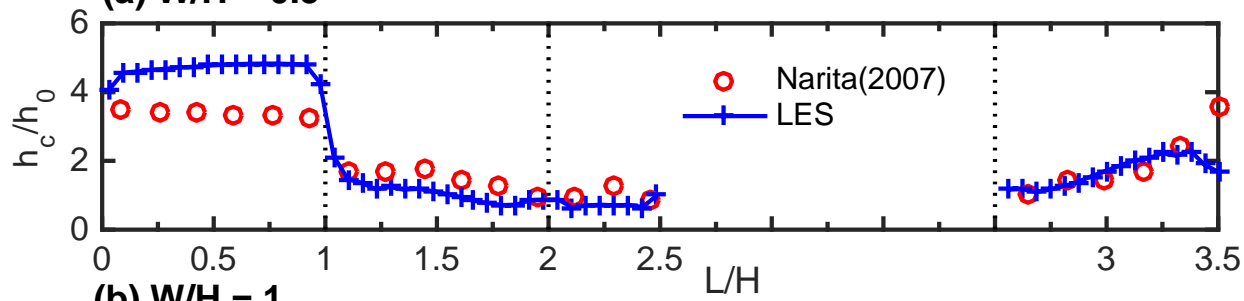

(b) $\mathrm{W} / \mathrm{H}=1$

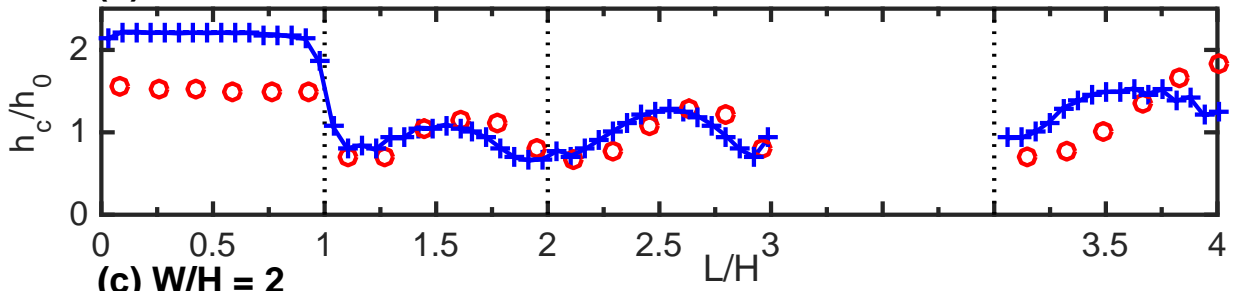

(c) $\mathrm{W} / \mathrm{H}=2$

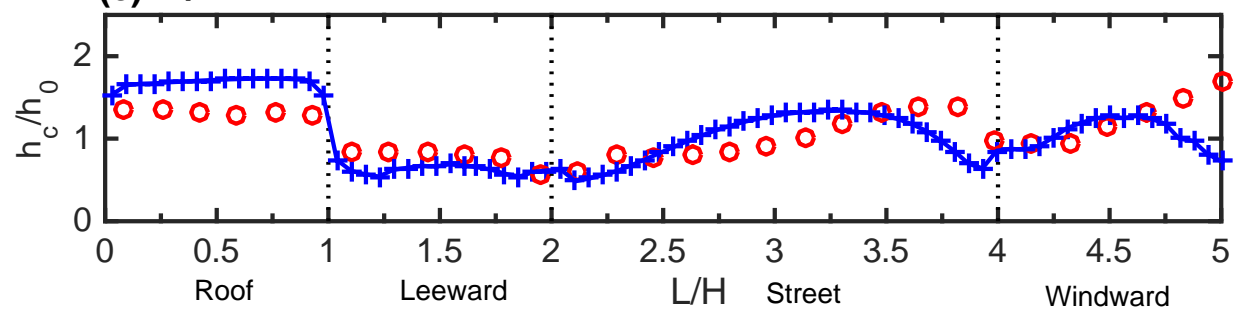

Fig. 4. The normalized mass transfer coefficient for different positions across the canyon. $L$ is the path length along the interface, and a unit $L / H$ is the length of the dotted line indicated in Fig. 1 for case $\mathrm{W} / \mathrm{H}=2$ as an example. The white space with no data for the cases in (a) and (b) does not reflect a data gap, but the fact that the street widths are shorter in these cases compared to the case in (c), which we adopt to fix the overall width of the figure.

Larger discrepancy between the observations and LES occurs near the top of the windward facet and on the roof, which can have two possible reasons. One potential reason for this larger discrepancy is the difference in the dominant drag mechanism: while pressure drag dominates at the vertical wall, the viscous drag dominates over the roof [66]. Another reason is related to our inability to match the experimental inflow 
conditions in LES exactly, as shown in Figure 5. The inflow vertical profiles of the normalized mean streamwise velocity and turbulent intensity (TI) at the upstream of location $x=0$ are shown in Figure 5. The mean velocity in both LES and experiment is normalized by its value at $z=H$, while the TI is computed locally. The mass transfer from the roof surface and upper part of the front/windward wall are more dependent on the inflow profile (mean velocity as well as turbulence intensity) than the bottom and the leeward faces. To test the sensitivity of the mass transfer for the different faces to inflow conditions, another test was conducted also assuming a fully periodic domain but without the long extension. This implies an infinite array of ribs, and is further removed from the actual setup in the wind tunnel. The results from this test (not shown here) indicate that while the absolute value of the error defined as $\left|\left(h_{L E S}-h_{E x p}\right) / h_{E x p}\right|$ remained similar for the leeward and bottom faces, the errors on the front and top faces were 3-5 times larger compared to the values presented in Table 1, which correspond to the basic setup. This further confirms the importance of characterizing the inflow in experiments accurately and reporting it in the associated paper to allow the data to be used for model validation, and supports our explanation that the higher discrepancy in the upper part of the windward facet and on the roof are related to a mismatch in the inflow. 


\begin{tabular}{lccccc} 
& Leeward & Street & Windward & Roof & Average \\
\hline $\boldsymbol{W} / \boldsymbol{H}=\mathbf{1} / \mathbf{2}$ & 18.2 & 15.5 & 20.3 & 42.3 & 25.5 \\
$\boldsymbol{W} / \boldsymbol{H}=\mathbf{1}$ & 11.1 & 12.0 & 30.2 & 35.5 & 22.5 \\
$\boldsymbol{W} / \boldsymbol{H}=\mathbf{2}$ & 20.2 & 22.5 & 17.4 & 27.8 & 22.0 \\
\hline
\end{tabular}

Table 1 . The absolute percentage deviation $(\%),\left|\left(h_{L E S}-h_{E x p}\right) / h_{E x p}\right| \times 100$, of the averaged transfer coefficient over each facet and all facets combined.
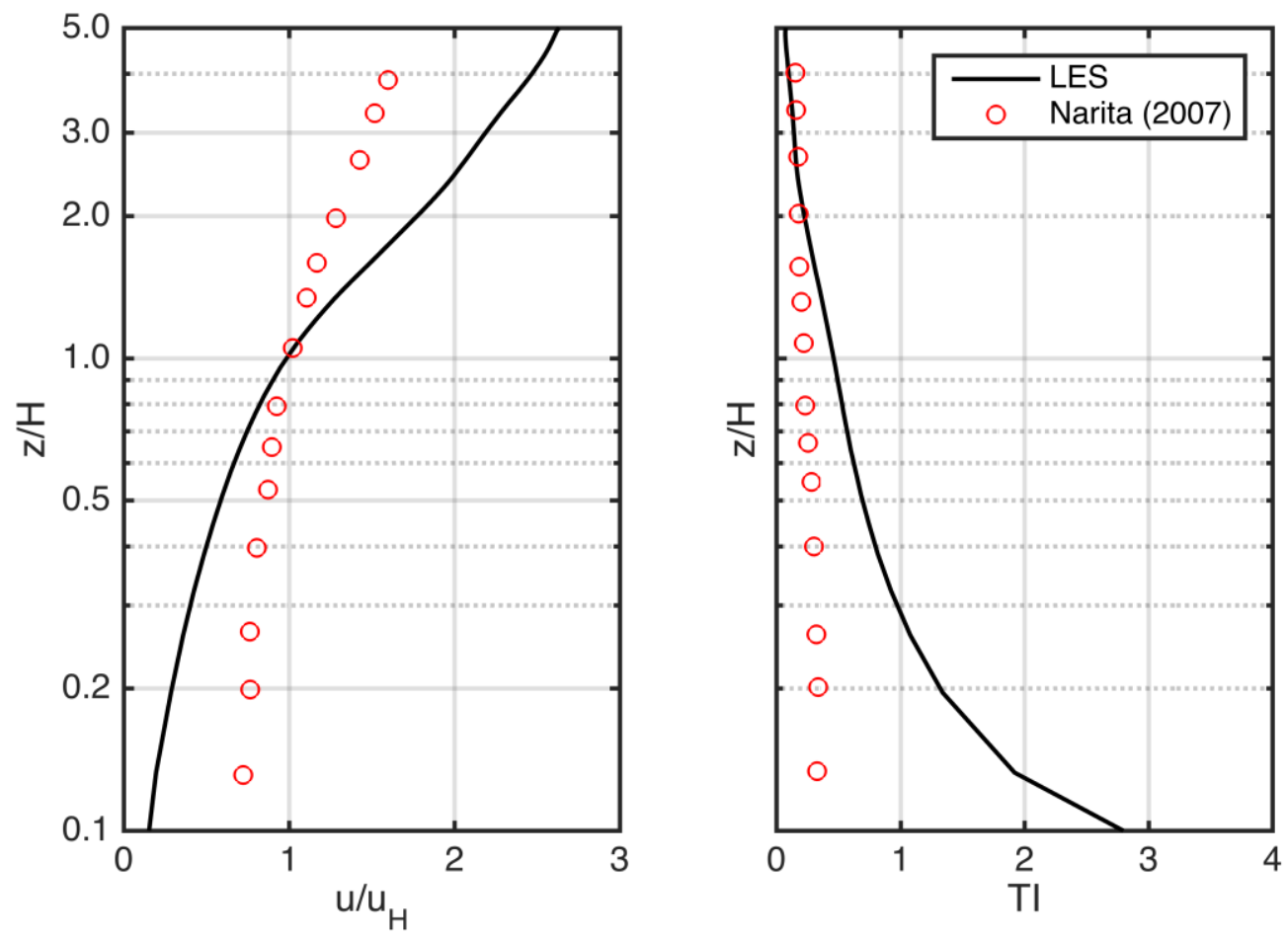

Fig. 5 The comparison between the mean streamwise velocity and turbulent intensity TI at the inflow section between LES and experiment. 


\section{Facet-averaged heat transfer from a cube compared to a wind tunnel study}

\subsection{Experimental set-up of heat transfer from a single cube}

The turbulent forced convective heat transfer over a wall-mounted cube at relatively low Reynolds number has been quite extensively studied as discussed in the introduction. In particular, we will focus on the study by Nakamura et al. [22] since their experiment was conducted at a relatively high $\operatorname{Re}$ - from 4,200 to 33,000 - despite the fact that is remains orders of magnitude lower than for real buildings. Furthermore, relations between $N u$ and $R e$ for different faces of the cube were proposed in that study, and they will be useful for our comparisons. In this experiment, a copper cube was heated by an embedded heater to maintain the surface temperature approximately constant (within $\pm 0.5^{\circ} \mathrm{C}$ ). The cube, with a dimension of $30 \mathrm{~mm}$, was placed in a low-speed wind tunnel of $4 \mathrm{~m}$ height, $3 \mathrm{~m}$

width, and $8 \mathrm{~m}$ length. A turbulent boundary layer is achieved by placing a horizontal circular cylinder $500 \mathrm{~mm}$ upstream from the cube to act as a trip. The diameter of the circular cylinder is $10 \mathrm{~mm}$ and the boundary layer depth to cube height ratio varies from 1.5 to 1.83 . A temperature difference of approximately $10^{\circ} \mathrm{C}$ is maintained between the surface of the cube and the air temperature. $R e$, defined based on the cube height and the bulk velocity upstream of the cube, was varied to assess how it is related to $N u$. 


\subsection{Numerical model of heat transfer from a single cube}

For all simulations in this section, a horizontally periodic domain is used. Figure 6 is the schematic drawing of the setup of the numerical simulation. 30 grid points are used along each side of the cube. The domain height is $4 H$, where $H$ is dimension of the cube. The upper boundary condition is impermeable with a free-slip for momentum and zero-gradient (no flux) for temperature. Five different simulations were performed at different Reynolds number in our LES by varying the horizontal pressure forcing, which is equivalent to changing the bulk velocity in the inflow. The Reynolds number is defined as $R e=U H / v$, where $U$ is the free stream velocity in the wind tunnel. The LES velocity used in $R e$ is taken at the location $(x, z)=(0,1.5 \mathrm{H})$, which provides a reasonable match to the experimental definition. Notice that in the LES setup the wall model defines an inner scale (since we are using a smooth-wall roughness length parameterization that depends on $v$ ), and the nominal $R e$ of the simulations can therefore be determined; viscous stresses are neglected in the numerical integration of the momentum and scalar equations. 


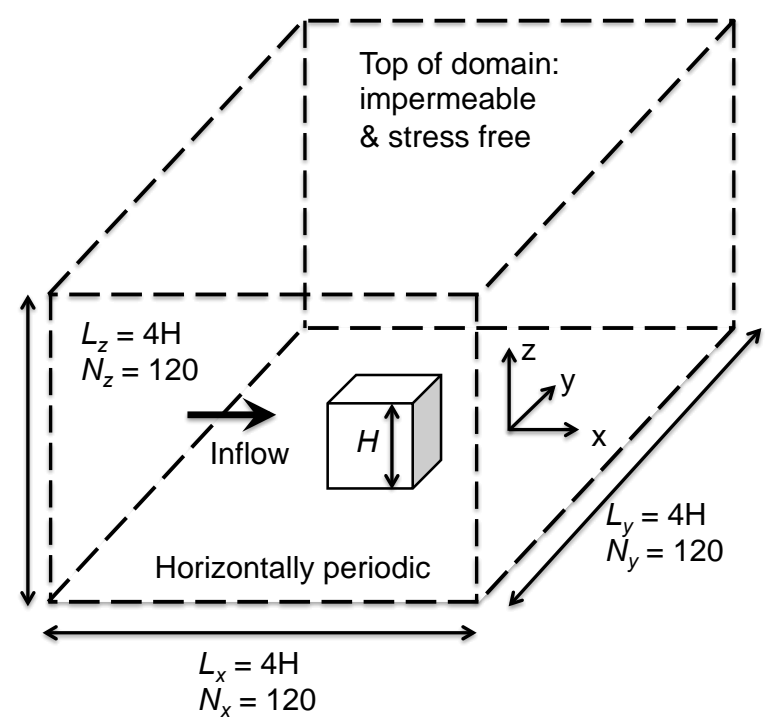

Fig.6. Schematic drawing of the setup of the numerical simulation. A heated cube of size $H$ is placed in the middle of the domain. The grid consists of $120^{3}$ nodes, and the domain size is $L_{x}=L_{y}=L_{z}=4 H$.

For all simulated cases, a constant temperature wall boundary condition is implemented in the wall model. All cases were simulated for a total of 100 eddy turnover times, defined as $L_{z} / u *$ (this corresponds to 400 eddy turnover times defined based on the cube scale). After a transient of 50 eddy turnovers, all time-averaged statistics reported were computed using the last 50 eddy turnovers times.

Figure 7(a) shows a vertical $x$ - $z$ transect along $y=2 H$ (middle of the cube), where both the contour of temperature deviation from the inflow temperature, defined as $\left(\theta-\theta_{\mathrm{i}}\right) / \theta_{\mathrm{i}}$, and the velocity streamlines are shown. Similarly, figure 7(b) is a horizontal transect at $z=0.015 H$ (near the floor). The temperature deviation contours depict large spatial 
gradients around the cube. The separation near $z=H / 2$, and the reattachment zone near the lower corner of the front face of the cube (figure 7(a)) compare well with experimental visualizations $[22,67]$. The separation zone and the two counter-rotating vortices shown in figure 7(b) near the rear face are also some well-known features of flow around a single cube, as seen for example in flow visualizations in Nakamura et al. [22] and Martinuzzi and Tropea [67].
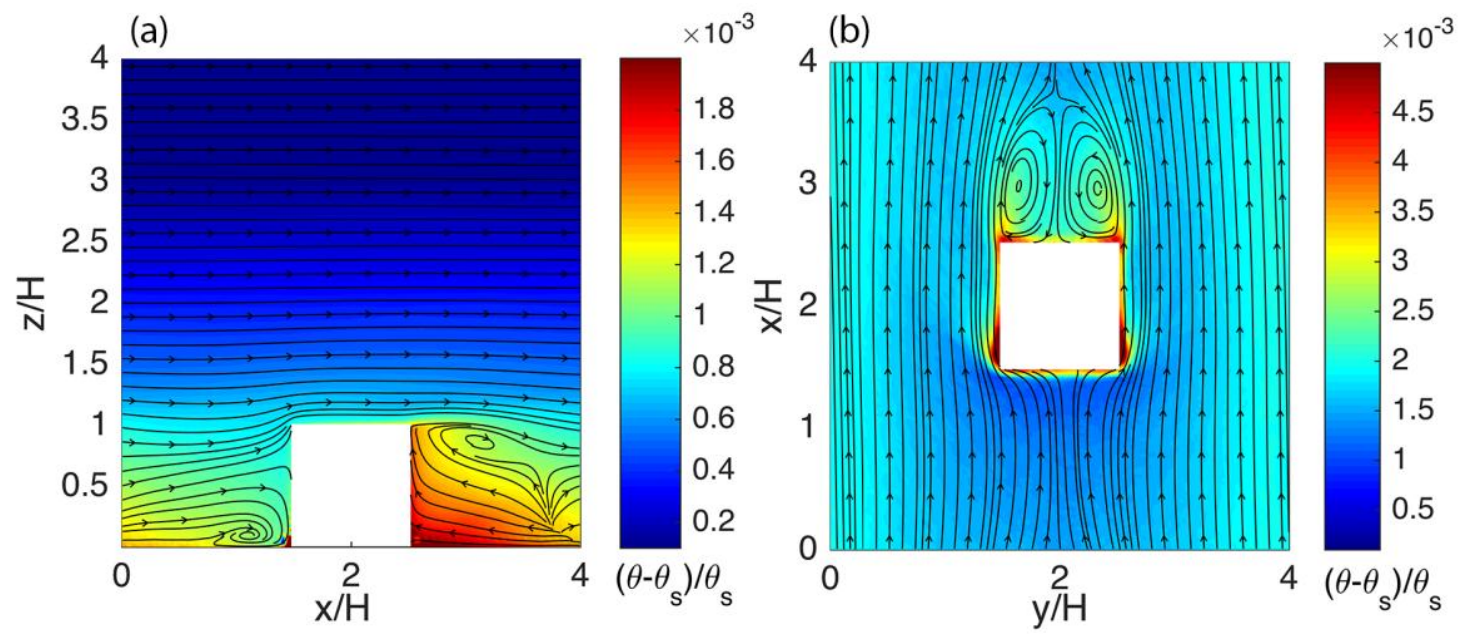

Fig.7. Mean flow field (streamlines) and contour plot of the temperature deviation from $\theta_{\text {inflow }}$ along (a) a vertical $x-z$ plane at $y=2 H$; and (b) a horizontal plane close to the floor at $z=0.015 H$.

The Nu-Re relation obtained from experimental measurements of Nakamura et al. [22] follow the classic power law

$$
N u=a R e^{m},
$$

the coefficients of which are given in Table 2. Due to the difference in $R e$, these 
experimental $\mathrm{Nu}$-Re relations of Nakamura et al. are extrapolated to the $R e$ of the LES for comparison. This ignores the well-known dependence of $m$ on $R e$, a caveat we will revisit in the next section. However, this approach was necessary since reducing our $R e$ further to match the experiment would place our first grid point in the viscous or buffer layers and preclude us from testing the wall-modeled LES configurations that we aim to use for full-scale (real-world) applications.

\begin{tabular}{ccc}
\hline & $\boldsymbol{a}$ & $\boldsymbol{m}$ \\
\hline front & 0.71 & 0.52 \\
side & 0.12 & 0.70 \\
rear & 0.11 & 0.67 \\
top & 0.071 & 0.74 \\
cube average & 0.138 & 0.68 \\
\hline
\end{tabular}

Table 2. The coefficients and exponents in Eq.(3) as determined in Nakamura et al. [22].

Figure 8(a) shows the comparisons between the relations proposed by Nakamura et al. [22], extrapolated to the LES Re, for the averaged $N u$ on different facets and the LES results. Although these experimental relationships were found at $R e$ orders of magnitude smaller, the match between predicted values according to Eq.(3) and those obtained from LES is in fact reasonable. The front and leeward faces show higher errors than the other faces, but errors cancel out and cube-averaged fluxes match quite well. This can be 
interpreted either as giving confidence in the performance of LES, or alternatively in the applicability of extrapolations from low $R e$ studies to the higher $R e$ flows in the real-world. Figure 8(b) shows that the ratio of deviation $R_{d}$ defined as:

$$
R_{d}=N u_{L E S} / N u_{E x p},
$$

where the experimental results are the values predicted from Eq.(3) and table 2, at different $R e$. Except for the front face which is excluded from this comparison, exchanges from the other faces remain within $50 \%$ of the measurements. The most likely reason why the front face deviates the most from the experimental result is that the experimental flow over that face could still be in a regime of laminar or transitional flow. This is strongly suggested by the small experimental exponent, 0.52 , which is considerably lower than that expected in turbulent flows, and rather very close to the 0.5 limit expected for laminar flows [68]. In addition, the turbulent boundary layer depth in the experiment is $1.5 \delta / H$, which is different than the fully developed one in LES of $4 \delta / H$.
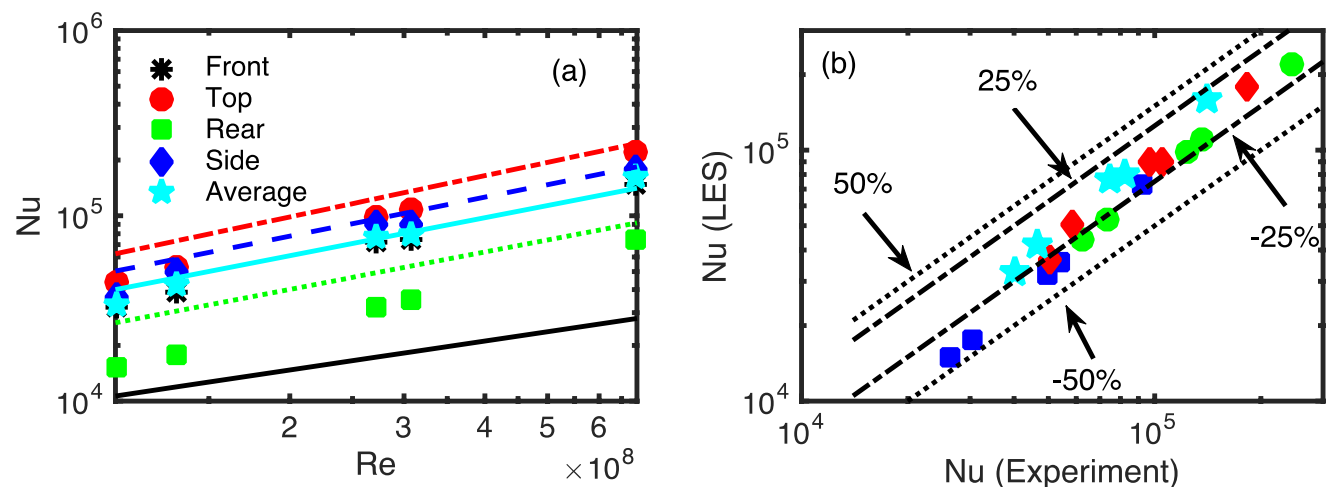

Fig.8.(a): $\mathrm{Nu}$-Re relation for different faces using empirical results from Nakamura et al.[22] i.e. using $m$ and $a$ from Table 2 and extrapolating to the Re of the LES. (b):Nusselt number of the 
experiment vs. that from LES. The black lines denote the quantities $N u_{\exp }\left(1+R_{d}\right)$, where $R_{d}= \pm 25$ and $\pm 50 \%$. The front face is excluded in (b) since its errors are much higher due to the Re discrepancy.

It is often of practical interest to use the cube-averaged or facet-averaged value of the heat transfer coefficient when considering the bulk heat exchange between a building envelope and the surrounding air, despite the high spatial variability. Figure 9(a) shows the contours of the heat transfer coefficient normalized by the cube average. Only one side-face is shown because of symmetry. Large deviations from the cube-averaged value occur on the edges as expected. The spatial variation at the intersections between front, top and rear faces is the most prominent. Figure 9(b) depicts the heat transfer coefficient normalized by the respective face-averaged values. Despite the large spatial variability at the intersections between difference faces, the cyan contour of value 1.1 indicates that the deviation over a large area of each face is only moderate. This implies that for practical applications, point-measured values in the center of a facet or numerically-determined face-averaged values give good estimates of the transfer over larger portions of each facet, despite some loss of information on the higher values near the corners. However, cube-averaged values should not be applied to individual facets. The contour plots in figure 9 also compare well qualitatively with results in the experiments of Nakamura et al. [22]. 
The wall friction velocity $u *$ and temperature scale $\theta *$, where $\quad *=q_{0} /\left(u_{*} c p\right)$, are shown in figure 10 (a) and (b) respectively. The spatial variability patterns of $u *$ are strongly correlated with those of $h_{c}$, indicating that the friction velocity has a strong impact on heat transfer as expected. The patterns of $\theta *$ on the other hand are distinct, with strong heat exchange near the bottom of the all faces due to the horseshoe vortex depicted in figure 7.
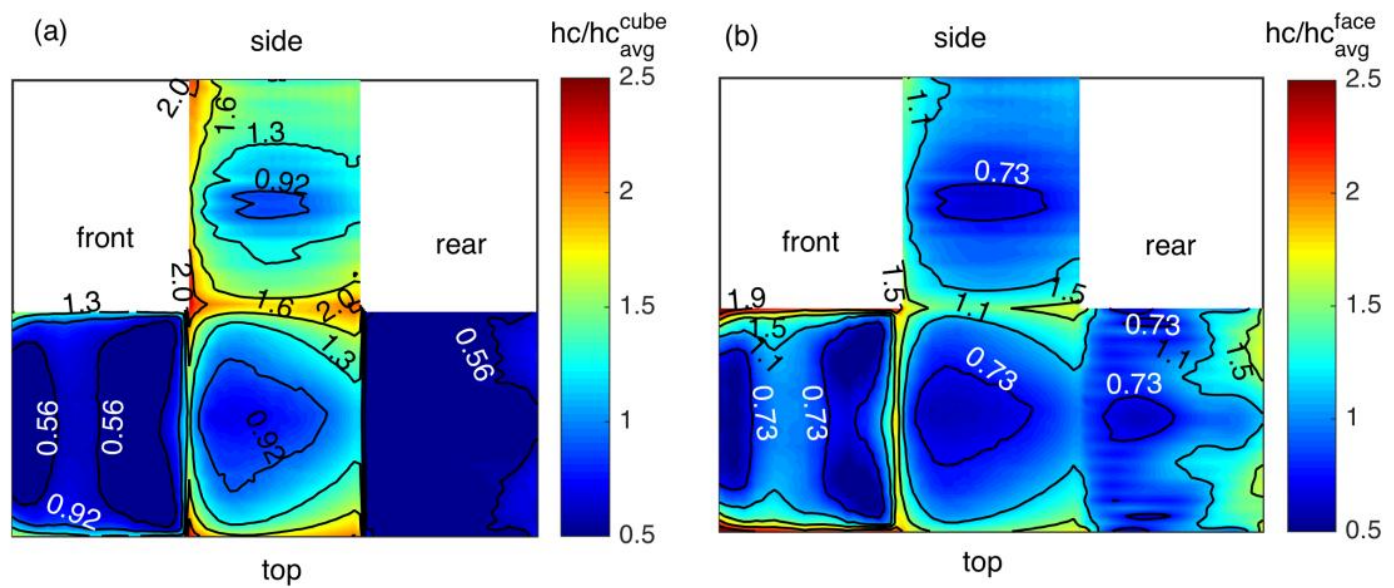

Fig.9. (a): Local heat transfer coefficient normalized by the cube-averaged value on all four facets.

(b): Local heat transfer coefficient normalized by each facet average value. 

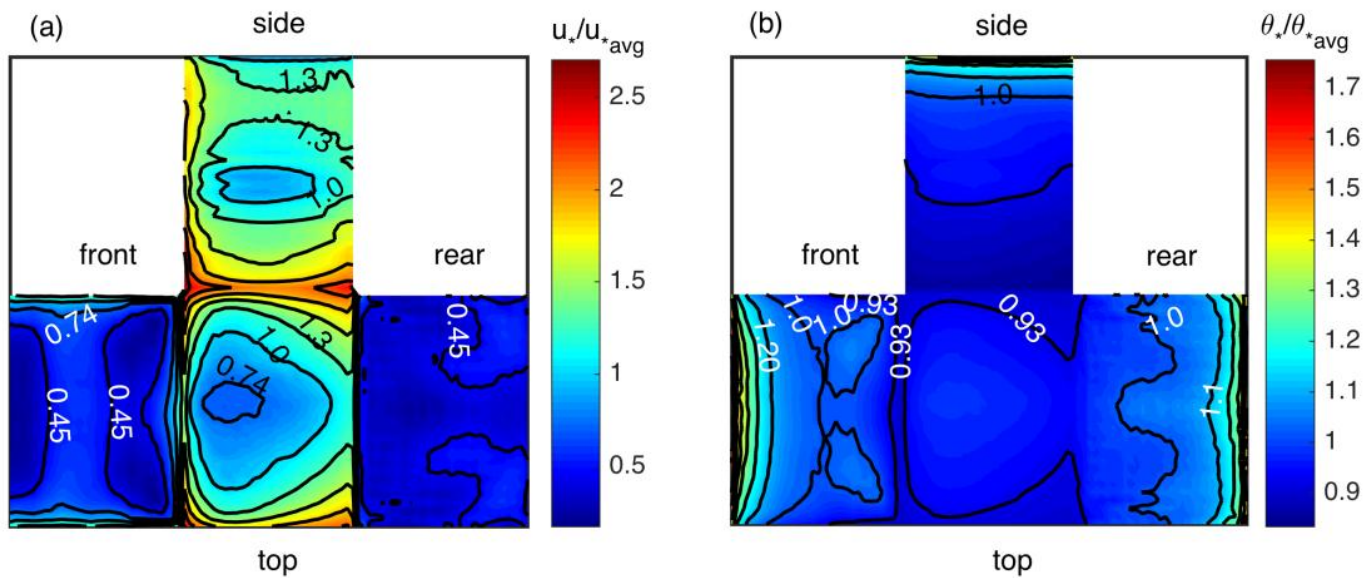

Fig.10. (a): Spatial distribution of the wall friction velocity $u *$ normalized by the cube average value. (b): Spatial distribution of the wall temperature scale $\theta *$ normalized by cube average value.

Separate sensitivity tests with varying domain heights of $1.7 H$ and $3 H$ were also conducted and yielded markedly different results due to the increased flow blockage resulting in higher velocities around the cube. As shown in Table 3, the shorter domains result in higher $\mathrm{Nu}$ as a consequence of these higher velocities. The much smaller difference between $3 \mathrm{H}$ and $4 H$ compared to $1.7 H$ and $4 H$ nevertheless indicated that convergence occurs when $L_{z} \approx 4 H$.

Percentage difference between surface averaged $N u$ compared to case $L_{z}=4 H$

\begin{tabular}{llllll}
\hline $\boldsymbol{L}_{z}$ & Front & Top & Rear & Side & Average \\
\hline $\mathbf{1 . 7 H}$ & +43.8 & +54.1 & +34.5 & +15.0 & +32.5 \\
$\mathbf{3 H}$ & +10.8 & +5.60 & +6.76 & +6.78 & +7.34 \\
\hline
\end{tabular}

Table 3 Percentage difference between surface averaged $N u$ compared to case $L_{z}=4 H$ 


\section{Comparison to full-scale field measurements}

Field measurements of heat transfer coefficients provide valuable information to evaluate high-Re numerical models with minimal discrepancy in the Reynolds number. We considered the measurement performed by Hagishima et al. [69] in detail for comparison. This outdoor measurement campaign was conducted over two sites: one was on a building roof, and the other on a vertical wall of a cubical extension mounted on a roof. We selected the building roof case for comparison, in which there is a better similarity in the setup between our numerical simulation and the field experiment. The roof surface energy balance equation, together with the temperature difference between the building surface and air temperature measurement, were used in the experiment to calculate the convective heat transfer coefficient $h_{c}$. The temperature and wind speed measurements on the roof were positioned at about $10 \%$ and $6 \%$ of the height of the building respectively. The general $\mathrm{Nu}$-Re relation was deduced from the experimental data and found to follow the power law relation

$$
N u=0.023 \operatorname{Re}^{0.891}
$$

with R-square value of 0.964 , irrespective of wind direction variability. The length scale in the Reynolds number is defined as the length from the roof edge considering the wind direction, while the velocity scale is $u_{0}=\sqrt{\overline{u^{2}+v^{2}+w^{2}}}$, with the wind components measured by the anemometers.

For the comparison between these field measurements and the LES in terms of the fitted 
relation between the Nusselt and Reynolds numbers, we estimate the Reynolds number based on the same definition of the characteristic length and velocity scales used by Hagishima et al. [69]. The same five sets of simulations presented in section 4 are used to estimate the $\mathrm{Nu}$-Re relation. The $h_{c}$ on the building roof is spatially variable as we showed in previous sections; this affects the field experimental results fitted from measurements at a few points. For accurate comparison, we extract the $h_{c}$ from the LES roof at the same locations where Hagishima et al. acquired measurements on the experimental roof. Figure 11 depicts the distribution of the exponent $m$ and coefficient $a$ in $N u=a R e^{m}$, found from linear-regression of the LES results at different $R e$ over the roof facet. The red marks denote where the experimental measuring points were positioned, approximately. On average, the spatial variation of the exponent $m$ is about $11 \%$, while a much greater variation is seen in the coefficient $a$, the values of which varied by one order of magnitude.

From the roof-averaged LES results and the ones averaged over the 4 experimental points, we respectively obtain

$$
N u_{\text {LES }}^{\text {roof-average }}=0.013 R e_{L E S}^{0.88} \quad, \quad N u_{L E S}^{4 \text { points-average }}=0.075 R e_{\text {LES }}{ }^{0.88} .
$$

The strong similarity in the exponent values in Eq.(3) and Eq.(3) indicates that our wall-modeled LES is able to capture the change in heat transfer coefficient well even as the wind speed (i.e. $R e)$ varies. The LES values of $a(0.013$ and 0.075$)$ bracket the experimental value (0.023). We do not anticipate being able to exactly capture the 
experimental value of $a$, as well as we capture $m$, for several reasons including:

1. Setup conditions in the field experiment and the LES cannot be exactly matched, and $a$ is highly sensitive to these conditions unlike $m$. For example, according to a report by Hagishima et al. [69], the $0.25 \mathrm{~m}$ protrusion around the building edge induces separation and backflow. The measuring height was $0.60 \mathrm{~m}$ above the roof-top but the wake caused by these intrusions can affect the exact magnitude of heat transfer reflected in $a$ (but not its scaling with $R e$ reflected by $m$ ).

2. The wall-model imposes a thermal roughness length in LES by assuming a smooth wall, but the actual smoothness of the roof used in the Hagishima et al. study is not characterized. Some building walls could very well be transitionally or hydrodynamically rough such that the actual roughness length $z o$ of these surfaces is needed to match $a$, although we point out that this would have also caused discrepancy in $m$.

Therefore, the LES can be expected to quantitatively predict the scaling represented by $m$ in the relation between the wind speed and forced convective heat transfer with high accuracy, but the exact magnitude of $h_{c}$ for a given wall also requires matching $a$ and is highly dependent on fine details such as wall texture and material, and surrounding obstructions. 

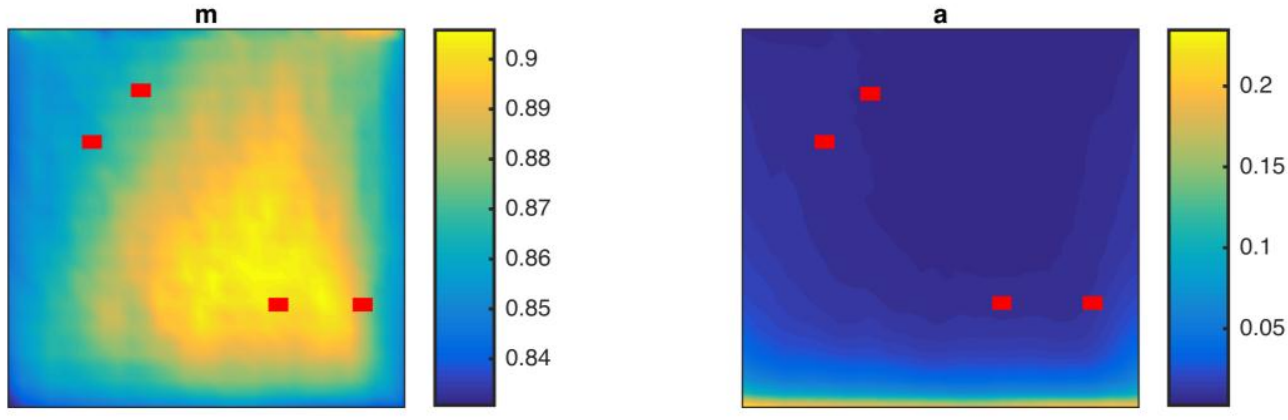

Fig.11. Spatial distribution of the best-fit results of $m$ and $a$ on the building roof, where the wind is blowing from bottom to top of the figure. The red dots are the location of the experimental measurements of Hagishima et al. [69].

Table 4 gives a summary of results from other field experiments that attempt to relate the heat transfer to change in wind velocity (i.e. $\mathrm{Nu}$ - Re relation). Although the experimental conditions and measurement techniques vary across these campaigns, and certainly discrepancies exist among them, there seems to be a consistent power law relation between the forced convective heat transfer and the wind speed with an exponent in the range of $0.67-0.89$. The wall-modeled LES considered in this paper is shown to give results that consistently fall within the experimental range. All of the exponents are $<1$, suggesting that the flow over the surfaces is not in the fully rough regime where the Stanton number would become independent of $R e$. 


\begin{tabular}{|c|c|c|}
\hline \multicolumn{2}{|r|}{ Experimental } & ES \\
\hline Emmel et al.[30] & 0.85 (Roof) & 0.88 \\
\hline Clear et al.[32] & 0.8 (Roof) & 0.88 \\
\hline \multirow[t]{2}{*}{ Yazdamian and Klems[31] } & 0.89 (Windward, low-rise building) & 0.89 \\
\hline & 0.671 (Leeward, low-rise building) & 0.90 \\
\hline
\end{tabular}

Table 4. The exponent in the $\mathrm{Nu}$ - $\mathrm{Re}$ relation for different experiments and corresponding values from LES.

\section{Discussion and Conclusions}

This study assessed the capability of the wall-modeled LES approach to capture the physics of forced convective heat/mass transfer between the surfaces of buildings and the atmosphere. Through detailed comparisons to both wind-tunnel studies and field experiment, we have shown that our LES is able to reasonably predict i) the spatial variation of the heat/mass transfer coefficient over the different facets of $2 \mathrm{D}$ ribs; ii) the average Nusselt number for a single cube (with larger discrepancy relative to measurements over the windward face very likely related to the $R e$ discrepancy); and iii) the power law relation between the Nusselt and Reynolds numbers compared to field measurements. The excellent match of the power law exponent $m$ is largely attributable to the dynamic wall model we proposed and implemented here.

Returning to the motivating question we asked: "are the errors resulting from the parameterization of unresolved scales (wall and subgrid scale models) in LES larger or 
smaller than the errors involved in extrapolating from low-Re approaches (DNS or wind tunnels) to high-Re real world flows, for scalar transfer problems?", the overall conclusion from out study indicates that the LES, despite its inherent parameterizations, is more suitable for studying real-world buildings:

1) Wind-tunnel studies result in $N u \sim R e^{0.52-0.74}$, a significantly lower exponent range than the $\approx 0.9$ observed in field measurements and LES. This is consistent with the expected trend of a lower $m$ when $R e$ is lower, and suggests that the low-Re effects in the wind tunnel are biasing the findings and would make them not suitable for extrapolation to the real-world (yet as mentioned in the introduction some current models rely on such coefficients empirically determined from water channel studies from 1924 [14]). As such, when LES-wind tunnel discrepancies arise, it seem more likely that the errors are related to the extrapolation of wind tunnel $\mathrm{Nu}$-Re relations outside their range of validity.

2) There is a strong sensitivity of the heat transfer exchange coefficient to inflow conditions, and the inflow is wind tunnel studies (or many simulations for that matter) do not represent realistic upwind conditions in the real world.

For building models and urban microclimate models that often use averaged value for modeling turbulent heat exchange, based on our simulation results, the use of facet-averaged values seem to be appropriate, but the relatively large differences among different facets preclude the use of a single coefficient for the whole building since this 
would not capture the large facet-to-facet variations. In addition, we have documented (not surprisingly) that it is important in numerical simulation like LES to match the experimental inflow conditions, especially for the windward faces that are affected the most. For future experimental studies in wind tunnels or field experiments, details such as the inflow profiles in a wind tunnel, measuring positions of wind and temperature, and wind directions should be included so that further validation studies can be conducted with more details of the experimental setup. For the types of numerical experiments considered here, the suitable domain height should be greater than 4 times the height of the obstacle. Another point to note is that the exponent $m$ in $N u \sim R e^{m}$ being close to 1.0 (both in building-scale field measurement and LES) is a manifestation of approaching the fully rough limit[27], in which the Stanton number is independent of Re. However, this limit is not reached suggesting that transitional effects persist. This should not be confused with the building canopy scale flow, which is clearly in the fully rough regime.

Going forward, the results gives us confidence in the capability of LES and the potential for using the technique to develop a better understanding of coupled scalar and momentum transfer at high-Re over complex topographies, and to formulate improved spatially-averaged surface exchange models to be used in coarse atmospheric models (weather or climate) where the buildings cannot be resolved. 


\section{Acknowledgement}

This study was funded by the US National Science Foundation's Sustainability Research Network Cooperative Agreement \# 1444758 and Water Sustainability and Climate

program Grant \# CBET-1058027. The simulations were performed on the supercomputing clusters of the National Center for Atmospheric Research through project P36861020. W.A. was supported by the Army Research Office Environmental Sciences Directorate (Grant \# W911NF-15-1-0231; PM: Dr. J. Parker ).

\section{References}

[1] M. Mirsadeghi, D. Cóstola, B. Blocken, J.L.M. Hensen, Review of external convective heat transfer coefficient models in building energy simulation programs: Implementation and uncertainty, Applied Thermal Engineering. 56 (2013) 134-151.

[2] T. Defraeye, B. Blocken, J. Carmeliet, Convective heat transfer coefficients for exterior building surfaces: Existing correlations and CFD modelling, Energy Conversion and Management. 52 (2011) 512-522. doi:10.1016/j.enconman.2010.07.026.

[3] J.A. Palyvos, A survey of wind convection coefficient correlations for building envelope energy systems' modeling, Applied Thermal Engineering. 28 (2008) 801-808. doi:10.1016/j.applthermaleng.2007.12.005.

[4] D. Li, E. Bou-Zeid, Quality and sensitivity of high-resolution numerical simulation of urban heat islands, Environ. Res. Lett. 9 (2014).

[5] T.H. Chilton, A.P. Colburn, Mass Transfer (Absorption) Coefficients Prediction from Data on Heat Transfer and Fluid Friction, Ind. Eng. Chem. 26 (1934) 1183-1187. doi:10.1021/ie50299a012.

[6] B. Blocken, J. Carmeliet, The influence of the wind-blocking effect by a building on its wind-driven rain exposure, Journal of Wind Engineering and Industrial Aerodynamics. 94 (2006) 101-127. 
[7] T. Sun, E. Bou-Zeid, Z.-H. Wang, E. Zerba, G.-H. Ni, Hydrometeorological determinants of green roof performance via a vertically-resolved model for heat and water transport, Building and Environment. 60 (2013) 211-224. doi:10.1016/j.buildenv.2012.10.018.

[8] T. Sun, E. Bou-Zeid, G.-H. Ni, To irrigate or not to irrigate: Analysis of green roof performance via a vertically-resolved hygrothermal model, Building and Environment. 73 (2014) 127-137. doi:10.1016/j.buildenv.2013.12.004.

[9] V. Masson, A physically-based scheme for the urban energy budget in atmospheric models, Boundary-Layer Meteorol. 94 (2000) 357-397. doi:10.1023/A:1002463829265.

[10] Z.-H. Wang, E. Bou-Zeid, J.A. Smith, A coupled energy transport and hydrological model for urban canopies evaluated using a wireless sensor network, Quarterly Journal of the Royal Meteorological Society. 139 (2013) 1643-1657. doi:10.1002/qj.2032.

[11] C.S.B. Grimmond, M. Blackett, M.J. Best, J.J. Baik, S.E. Belcher, J. Beringer, et al., Initial results from Phase 2 of the international urban energy balance model comparison, International Journal of Climatology. 31 (2011) 244-272. doi:10.1002/joc.2227.

[12] C.S.B. Grimmond, T.R. Oke, D.G. Steyn, Urban Water Balance 1. A Model for Daily Totals, (1986).

[13] A. Hagishima, J. Tanimoto, K.I. Narita, Intercomparisons of experimental convective heat transfer coefficients and mass transfer coefficients of urban surfaces, Boundary-Layer Meteorol. 117 (2005) 551-576.

[14] W. Jürges, Der Wärmeübergang an einer ebenen Wand, 1924.

[15] I.P. Castro, H. Cheng, R. Reynolds, Turbulence Over Urban-type Roughness: Deductions from Wind-tunnel Measurements, Boundary-Layer Meteorol. 118 (2006) 109-131. doi:10.1007/s10546-005-5747-7.

[16] J. Jiménez, TURBULENT FLOWS OVER ROUGH WALLS, Http://Dx.Doi.org/10.1146/Annurev.Fluid.36.050802.122103. 36 (2004) 173-196. doi:10.1146/annurev.fluid.36.050802.122103.

[17] D.A. Aliaga, J.P. Lamb, D.E. Klein, Convection heat transfer distributions over plates with square ribs from infrared thermography measurements, International Journal of Heat and Mass Transfer. 37 (1994) 363-374. doi:10.1016/0017-9310(94)90071-X.

[18] M.K. Chyu, V. Natarajan, Local heat/mass transfer distributions on the surface of a wall- mounted cube, Transections of the ASME. Journal of Heat Transfer. 113 (1991) 851-857. doi:10.1115/1.2911213. 
[19] T. Igarashi, Heat transfer from a square prism to an air stream, International Journal of Heat and Mass Transfer. 28 (1985) 175-181.

doi:10.1016/0017-9310(85)90019-5.

[20] E.R. Meinders, T.H. Van Der Meer, K. Hanjalić, Local convective heat transfer from an array of wall-mounted cubes, International Journal of Heat and Mass Transfer. 41 (1998) 335-346.

doi:10.1016/S0017-9310(97)00148-8.

[21] E.R. Meinders, K. Hanjalić, Vortex structure and heat transfer in turbulent flow over a wall-mounted matrix of cubes, International Journal of Heat and Mass Transfer. 20 (1999) 255-267. doi:10.1016/S0142-727X(99)00016-8.

[22] H. Nakamura, T. Igarashi, T. Tsutsui, Local heat transfer around a wall-mounted cube in the turbulent boundary layer, International Journal of Heat and Mass Transfer. 44 (2001) 3385-3395. doi:10.1016/S0017-9310(01)00009-6.

[23] K.I. Narita, Experimental study of the transfer velocity for urban surfaces with a water evaporation method, Boundary-Layer Meteorol. 122 (2007) 293-320.

[24] F. Pascheke, J.F. Barlow, A. Robins, Wind-tunnel Modelling of Dispersion from a Scalar Area Source in Urban-Like Roughness, Boundary-Layer Meteorol. 126 (2007) 103-124. doi:10.1007/s10546-007-9222-5.

[25] J.F. Barlow, I.N. Harman, S.E. Belcher, Scalar fluxes from urban street canyons. Part I: Laboratory simulation, Boundary-Layer Meteorol. 113 (2004) 369-385. doi:10.1007/s10546-004-6204-8.

[26] J.H. Lienhard, A Heat Transfer Textbook, Courier Corporation, 2013.

[27] R.L. Webb, E.R.G. Eckert, R.J. Goldstein, Heat transfer and friction in tubes with repeated-rib roughness, International Journal of Heat and Mass Transfer. 14 (1971) 601-617. doi:10.1016/0017-9310(71)90009-3.

[28] F.L. Test, R.C. Lessmann, A. Johary, Heat transfer during wind flow over rectangular bodies in the natural environment, Transections of the ASME. Journal of Heat Transfer. 103 (1981) 262-267. doi:10.1115/1.3244451.

[29] D.L. Loveday, A.H. Taki, Convective heat transfer coefficients at a plane surface on a full-scale building facade, International Journal of Heat and Mass Transfer. 39 (1996) 1729-1742. doi:10.1016/0017-9310(95)00268-5.

[30] M.G. Emmel, M.O. Abadie, N. Mendes, New external convective heat transfer coefficient correlations for isolated low-rise buildings, Energy and Buildings. 39 (2007) 335-342.

[31] M. Yazdanian, J.H. Klems, Measurement of the exterior convective film coefficient for windows in low-rise buildings, ASHRAE Transactions. 100 (1994) 1087-1096. 
[32] R.D. Clear, L. Gartland, F.C. Winkelmann, An empirical correlation for the outside convective air-film coefficient for horizontal roofs, Energy and Buildings. 35 (2003) 797-811.

[33] Y. Liu, D.J. Harris, Full-scale measurements of convective coefficient on external surface of a low-rise building in sheltered conditions, Building and Environment. 42 (2007) 2718-2736.

[34] S.B. Park, J.J. Baik, A large-eddy simulation study of thermal effects on turbulence coherent structures in and above a building array, J. Appl. Meteor. Climatol. 52 (2013) 1348-1365.

[35] V.B.L. Boppana, Z.-T. Xie, I.P. Castro, Large-Eddy Simulation of Heat Transfer from a Single Cube Mounted on a Very Rough Wall, Boundary-Layer Meteorol. 147 (2012) 347-368. doi:10.1007/s10546-012-9793-7.

[36] T. Defraeye, B. Blocken, J. Carmeliet, CFD simulation of heat transfer at surfaces of bluff bodies in turbulent boundary layers: Evaluation of a forced-convective temperature wall function for mixed convection, Journal of Wind Engineering and Industrial Aerodynamics. 104-106 (2012) 439446.

[37] J. Liu, J. Srebric, N. Yu, Numerical simulation of convective heat transfer coefficients at the external surfaces of building arrays immersed in a turbulent boundary layer, International Journal of Heat and Mass Transfer. 61 (2013) 209-225. doi:10.1016/j.ijheatmasstransfer.2013.02.005.

[38] S. Leonardi, P. Orlandi, R.J. Smalley, L. Djenidi, R.A. Antonia, Direct numerical simulations of turbulent channel flow with transverse square bars on one wall, Journal of Fluid Mechanics. 491 (n.d.) 229-238.

[39] S. Leonardi, L. Djenidi, P. Orlandi, R.A. Antonia, Heat transfer in a turublent channel flow with square bars and circular rods on one wall, Journal of Fluid Mechanics. 776 (n.d.) 512-530.

[40] O. Coceal, T.G. Thomas, I.P. Castro, S.E. Belcher, Mean Flow and Turbulence Statistics Over Groups of Urban-like Cubical Obstacles, Boundary-Layer Meteorol. 121 (2006) 491-519. doi:10.1007/s10546-006-9076-2.

[41] O. Coceal, T.G. Thomas, S.E. Belcher, Spatial Variability of Flow Statistics within Regular Building Arrays, Boundary-Layer Meteorol. 125 (2007) 537552. doi:10.1007/s10546-007-9206-5.

[42] S.B. Pope, Turbulent Flows, Cambridge University Press, 2000.

[43] D.B. Spalding, A new analytical expression for the drag of a flat plate valid for both the turbulent and laminar regimes, International Journal of Heat and Mass Transfer. 5 (1962) 1133-1138. doi:10.1016/0017-9310(62)90189-8. 
[44] C.H. Liu, T.N.H. Chung, Forced convective heat transfer over ribs at various separation, International Journal of Heat and Mass Transfer. 55 (2012) 51115119. doi:10.1016/j.ijheatmasstransfer.2012.05.012.

[45] M.G. Giometto, A. Christen, C. Meneveau, J. Fang, M. Krafczyk, M.B. Parlange, Spatial Characteristics of Roughness Sublayer Mean Flow and Turbulence Over a Realistic Urban Surface, Boundary-Layer Meteorol. (2016) 1-28. doi:10.1007/s10546-016-0157-6.

[46] B.E. Launder, On the Computation of Convective Heat-Transfer in Complex Turbulent Flows, Journal of Heat Transfer-Transactions of the Asme. 110 (1988) 1112-1128.

[47] J.C. Wyngaard, L.J. Peltier, S. Khanna, LES in the surface layer: Surface fluxes, scaling, and SGS modeling, J. Atmos. Sci. 55 (1998) 1733-1754. doi:10.1175/1520-0469(1998)055<1733:LITSLS>2.0.CO;2.

[48] W. Cabot, P. Moin, Approximate wall boundary conditions in the large-eddy simulation of high Reynolds number flow, Flow, Turbulence and Combustion. 63 (2000) 269-291. doi:10.1023/A:1009958917113.

[49] X.I.A. Yang, J. Sadique, R. Mittal, C. Meneveau, Integral wall model for large eddy simulations of wall-bounded turbulent flows, Physics of Fluids (1994-Present). 27 (2015) 025112. doi:10.1063/1.4908072.

[50] X. Yang, J. Sadique, R. Mittal, C. Meneveau, Exponential roughness layer and analytical model for turbulent bounday layer flow over rectangular-prism roughness elements, Journal of Fluid Mechanics. 789 (n.d.) $127-165$.

[51] T. Defraeye, B. Blocken, J. Carmeliet, An adjusted temperature wall function for turbulent forced convective heat transfer for bluff bodies in the atmospheric boundary layer, Building and Environment. 46 (2011) 21302141. doi:10.1016/j.buildenv.2011.04.013.

[52] S.B. Pope, Ten questions concerning the large-eddy simulation of turbulent flows, New Journal of Physics. 6 (2004) -35.

doi:10.1088/1367-2630/6/1/035.

[53] J. Slotnick, CFD Vision 2030 Study, 2014.

[54] S. Chester, C. Meneveau, M.B. Parlange, Modeling turbulent flow over fractal trees with renormalized numerical simulation, Journal of Computational Physics. 225 (2007) 427-448. doi:10.1016/j.jcp.2006.12.009.

[55] Y.H. Tseng, C. Meneveau, M.B. Parlange, Modeling flow around bluff bodies and predicting urban dispersion using large eddy simulation, Environmental Science \& Technology. 40 (2006) 2653-2662. doi:10.1021/es051708m. 
[56] V. Kumar, J. Kleissl, C. Meneveau, M.B. Parlange, Large-eddy simulation of a diurnal cycle of the atmospheric boundary layer: Atmospheric stability and scaling issues, Water Resources Research. 42 (2006) n/a-n/a. doi:10.1029/2005WR004651.

[57] S. Shah, E. Bou-Zeid, Very-Large-Scale Motions in the Atmospheric Boundary Layer Educed by Snapshot Proper Orthogonal Decomposition, Boundary-Layer Meteorol. 153 (2014) 355-387. doi:10.1007/s 10546-014-9950-2.

[58] Q. Li, E. Bou-Zeid, W. Anderson, The impact and treatment of the Gibbs phenomenon in immersed boundary method simulations of momentum and scalar transport

, Journal of Computational Physics. (n.d.).

[59] E. Bou-Zeid, C. Meneveau, M. Parlange, A scale-dependent Lagrangian dynamic model for large eddy simulation of complex turbulent flows, Physics of Fluids. 17 (2005). doi:10.1063/1.1839152.

[60] W. Anderson, Passive scalar roughness lengths for atmospheric boundary layer flow over complex, fractal topographies, Environ Fluid Mech. 13 (2013) 479-501. doi:10.1007/s10652-013-9272-9.

[61] B.A. Kader, A.M. Yaglom, Heat and mass transfer laws for fully turbulent wall flows, International Journal of Heat and Mass Transfer. 15 (1972) 2329-2351. doi:10.1016/0017-9310(72)90131-7.

[62] A.E. Perry, J.B. Bell, P.N. Joubert, Velocity and temperature profiles in adverse pressure gradient turbulent boundary layers, Journal of Fluid Mechanics. 25 (1966) 299-320. doi:10.1017/S0022112066001666.

[63] L. Temmerman, M.A. Leschziner, C.P. Mellen, J. Fröhlich, Investigation of wall-function approximations and subgrid-scale models in large eddy simulation of separated flow in a channel with streamwise periodic constrictions, International Journal of Heat and Mass Transfer. 24 (2003) 157-180. doi:10.1016/S0142-727X(02)00222-9.

[64] T.R. Oke, Boundary Layer Climates, Routledge, 1978.

[65] A.E. Perry, W.H. Schofield, P.N. Joubert, Rough wall turbulent boundary layers, Journal of Fluid Mechanics. 37 (1969) 383-413. doi:10.1017/S0022112069000619.

[66] S. Leonardi, I.P. Castro, Journal of Fluid Mechanics - Abstract - Channel flow over large cube roughness: a direct numerical simulation study, Journal of Fluid Mechanics. (2010).

[67] R. Martinuzzi, C. Tropea, The Flow Around Surface-Mounted, Prismatic Obstacles Placed in a Fully Developed Channel Flow (Data Bank Contribution), J. Fluids Eng. 115 (1993) 85-92. doi:10.1115/1.2910118. 
[68] F.P. Incropera, Introduction to Heat Transfer Fourth Edition Wie, Wiley, 2002.

[69] A. Hagishima, J. Tanimoto, Field measurements for estimating the convective heat transfer coefficient at building surfaces, Building and Environment. 38 (2003) 873-881. doi:10.1016/S0360-1323(03)00033-7. 